\title{
CLUE: An Observational Checklist for Usability Evaluation of Multimodal Games for Children Who Are Blind
}

\author{
Ticianne Darin \\ Instituto Universidade Virtual \\ Universidade Federal do Ceará \\ ticianne@virtual.ufc.br
}

\author{
Rossana Andrade \\ Depto. Ciência da Computação \\ Universidade Federal do Ceará \\ rossana@great.ufc.br
}

\author{
Jaime Sánchez \\ Depto. Ciência da Computação \\ Universidade do Chile \\ jsanchez@dcc.uchile.cl
}

\begin{abstract}
This paper summarizes the proposal and validation of a Checklist for Usability Evaluation of Multimodal Games for Children who are Blind (CLUE). CLUE was designed to assist researchers and practitioners in usability evaluation field studies involving people who are blind, addressing multiple aspects of gameplay and multimodality, including audio, graphics, and haptics. Overall, initial evidence indicates that the use of CLUE during user observation helps to raise a greater number of relevant usability issues than other methods such as interview and questionnaire.
\end{abstract}

\section{Author Keywords}

Usability evaluation; multimodal video games; audio-haptic interfaces; interaction modes; people who are blind.

\section{ACM Classification Keywords}

H.5.2. Information interfaces and presentation: User Interfaces (D2.2, H1.2, I3.6);

\section{INTRODUÇÃO}

Quando pessoas cegas jogam videogames multimodais (baseados em estímulos sonoros e hápticos, por exemplo), é fundamental garantir a usabilidade e proporcionar uma interface adequada às tarefas e necessidades dos usuários, além de uma interação eficiente e confortável. Esse aspecto é crucial nesse cenário porque jogos multimodais podem ser usados para desenvolver habilidades cognitivas em pessoas cegas [4]. Assim, problemas de usabilidade se tornam um obstáculo não somente para a interação, mas para o desenvolvimento cognitivo. Ao conduzir testes de usabilidade envolvendo pessoas cegas, os avaliadores devem ter em mente que os Métodos de Avaliação de Usabilidade (Usability Evaluation Methods - UEM) tradicionais são geralmente projetados para usuários sem deficiências [2] e que seu uso generalizado em diferentes contextos é controverso [3]. A multimodalidade adiciona mais complexidade a este cenário, uma vez que questões

\footnotetext{
Permission to make digital or hard copies of all or part of this work for personal or classroom use is granted without fee provided that copies are not made or distributed for profit or commercial advantage and that copies bear this notice and the full citation on the first page. Copyrights for components of this work owned by others than the author(s) must be honored. Abstracting with credit is permitted. To copy otherwise, or republish, to post on servers or to redistribute to lists, requires prior specific permission and/or a fee. Copyright 2018 SBC.
}

IHC 2018, Anais Estendidos do XVII Simpósio Brasileiro sobre Fatores Humanos em Sistemas Computacionais

Outubro 22-26, 2018, Belém, Brasil

Artigos Internacionais específicas diferenciam a avaliação de usabilidade multimodal da avaliação de interfaces de usuário tradicionais, como GUIs [1]. Além disso, quando os usuários cegos se tratam de crianças, a avaliação da usabilidade deve ser tratada com mais cuidado porque elas ainda estão construindo conceitos abstratos e não percebem objetos e atividades das mesma forma que adultos na mesma condição [9]. No entanto, a literatura pouco tem discutido sobre quais aspectos avaliar e como conduzir a avaliação de usabilidade de jogos multimodais envolvendo crianças cegas [5].

Com o objetivo de fomentar o conhecimento e a prática nessa área e compartilhar nossos achados com a comunidade brasileira, o presente artigo resume a pesquisa originalmente publicada em [7], que propõe uma ferramenta de observação para testes de usabilidade, o Checklist for Usability Evaluation of Multimodal Games for Children who are Blind (daqui em diante abreviado como CLUE). CLUE visa guiar a observação de crianças cegas interagindo com jogos multimodais, durante estudos de campo e testes em laboratório, ajudando a identificar problemas na interface multimodal e na interação com tais videogames. Sua principal contribuição é o suporte à análise focada dos elementos da interface multimodal durante a interação, revelando questões que podem afetar os objetivos de tais videogames para crianças cegas.

\section{METODOLOGIA}

A pesquisa exploratória conduzida para propor CLUE foi adaptada das diretrizes de Stufflebeam (2000) para o desenvolvimento de checklists de avaliação [10]. A metodologia consistiu em três etapas principais: (i) Definição de Checkpoints; (ii) Categorização de Checkpoints; e (iii) Avaliação do Checklist. Durante a primeira etapa, uma lista de checkpoints foi criada com base nos problemas de usabilidade mais comuns na interação de pessoas cegas com jogos multimodais (conforme Standard List of Usability Problems - SLUP [6]). Na segunda etapa, os checkpoints foram descritos e classificados de acordo com as modalidades normalmente presentes nesses jogos [1].

Finalmente, a terceira etapa ocorreu em duas fases principais. Primeiro, cinco especialistas analisaram os checkpoints e deram feedback por e-mails e entrevistas nãoestruturadas sobre a adequação e abrangência dos checkpoints candidatos. A segunda fase consistiu em sessões de testes de usabilidade, com o objetivo de obter impressões de potenciais usuários do checklist, ao envolvê-los em um 
teste de campo utilizando o instrumento. Assim, quatro avaliadores independentes conduziram sessões de avaliação com seis crianças com diagnósticos oftalmológicos diferentes (todos legalmente cegos), de 8 a 14 anos de idade, frequentando de 2 a 7 séries em escolas para alunos cegos.

\section{CLUE: VISÃO GERAL}

A versão mais recente do $\mathrm{CLUE}^{1}$ contém 40 checkpoints agrupados em 4 categorias: Gameplay (14 itens), Acoustics (11 itens), Haptics (12 itens) e Graphics (3 itens). Gameplay contém itens relacionados a aspectos gerais de usabilidade e jogabilidade. Acoustics agrega itens relacionados à compreensibilidade e adequação dos múltiplos tipos de sons utilizados na interface do usuário. Haptics contém checkpoints relacionados ao uso de técnicas de interação háptica e dispositivos com feedback tátil que podem afetar a interação do usuário com as entradas e saídas do jogo. Graphics contém itens relacionados aos aspectos visuais da interface do usuário.

Cada categoria contém, ainda, checkpoints relacionados ao sentimento e à satisfação do usuário em relação a aspectos específicos do jogo, modalidades e tipos de feedback fornecidos por cada modalidade. CLUE pode ser usado de forma modular, de acordo com o contexto e modalidades do jogo, pois as categorias são independentes. Por exemplo, somente se uma pessoa com baixa visão estiver participando dos testes, os avaliadores utilizarão a categoria Graphics. Além disso, CLUE sugere um formato para pré-avaliar a severidade dos problemas encontrados, bem como apontar em que tarefas ocorreram quais problemas de usabilidade.

\section{RESUMO DOS RESULTADOS DO ESTUDO DE CAMPO}

$O$ estudo de campo visava engajar especialistas no teste do CLUE em um ambiente real, para coletar feedback autêntico, o que foi útil para refiná-lo. Utilizando exclusivamente o CLUE, os avaliadores identificaram $66 \%$ dos problemas de usabilidade encontrados posteriormente pela análise de vídeo da interação, que é mais dispendiosa. CLUE identificou mais problemas que uma entrevista semiestruturada e um questionário pós-teste especializado, principalmente no caso de problemas de interação, feedback e satisfação do usuário. A pesquisa original [7] discute em maiores detalhes a análise de dados e os tipos e subcategorias de problemas de usabilidade identificados por cada UEM.

\section{CONCLUSÃO}

CLUE ajuda avaliadores a não esquecer critérios importantes durante a avaliação de jogos multimodais envolvendo crianças cegas, além de aumentar a objetividade e reprodutibilidade da avaliação. Encorajamos a comunidade a se unir aos nossos esforços, aplicando o CLUE em suas pesquisas e nos dando feedback para a evolução desse instrumento. Como trabalho futuro, CLUE será testado com adultos cegos e expandido para uso em aplicações diversas.

\section{REFERÊNCIAS}

1. Niels Bernsen, Laila Dybkjær: Multimodal usability. Springer, Berlin (2009).

2. Sambhavi Chandrashekar, Tony Stockman, Deborah Fels, and Rachel Benedyk: Using think aloud protocol with blind users: a case for inclusive usability evaluation methods. In: 8th international ACM SIGACCESS conference on Computers and accessibility. pp. 251-252. ACM (2006)

3. Parmit K. Chilana, Jacob O. Wobbrock, and Andrew J. Ko. 2010. Understanding usability practices in complex domains. In Proceedings of the SIGCHI Conference on Human Factors in Computing Systems (CHI '10). ACM, NY, USA, 2337-2346.

4. Erin C. Connors, Elizabeth R. Chrastil, Jaime Sánchez, and Lotfi B. Merabet. 2014. Action video game play and transfer of navigation and spatial cognition skills in adolescents who are blind. Frontiers in Human Neuroscience 8, 133.

5. Ticianne Darin, Jaime Sánchez, and Rossana Andrade. Dimensions for the design and evaluation of multimodal videogames for the cognition of people who are blind. 2015. In: Proceedings of the 14th Brazilian Symposium on Human Factors in Computing Systems. ACM. p. 20-29.

6. Ticianne Darin, Rossana Andrade, Lotfi Merabet, and Jaime Sánchez. 2017. Investigating the Mode in Multimodal Video Games: Usability Issues for Learners who are Blind. In: Proceedings of the CHI Conference Extended Abstracts on Human Factors in Computing Systems (CHI EA '17). ACM, NY, USA, 2487-2495.

7. Ticianne Darin, Rossana Andrade, Jaime Sánchez. 2018. CLUE: A Usability Evaluation Checklist for Multimodal Video Games Field Studies with Children Who Are Blind In: Proceedings of the 51th Annual Hawaii International Conference on Computer Science. IEEE. p. 245-254

8. Jaime Sánchez. 2003. Software Usability for Blind Children Questionnaire (SUBC). http:// dx.doi.org/10.13140/RG.2.2.36286.08003

9. Christopher Spencer, Kim Morsley, Simon Ungar, Emma Pike, and Mark Blades. (1992). Developing the blind child's cognition of the environment: the role of direct and map-given experience. Geofo-rum, 23(2), 191-197

10. Daniel Stufflebeam. (2000). Guidelines for developing evaluation checklists: the checklists development checklist (CDC). Kalamazoo, MI: The Evaluation Center

\footnotetext{
${ }^{1}$ Versão completa do CLUE: https://goo.gl/pWuKLk
} 